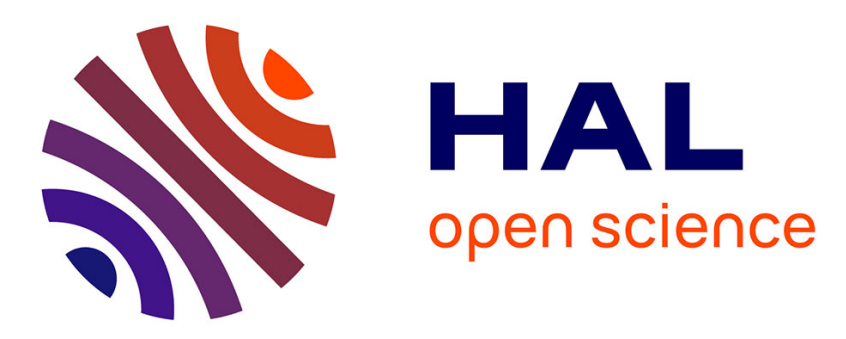

\title{
Nonlocal/coarse graining homogenization of linear elastic media with non-separated scales using least-square polynomial filters
}

\author{
Julien Yvonnet, Guy Bonnet
}

\section{- To cite this version:}

Julien Yvonnet, Guy Bonnet. Nonlocal/coarse graining homogenization of linear elastic media with non-separated scales using least-square polynomial filters. International Journal for Multiscale Computational Engineering, 2014, 12 (5), pp.375-395. 10.1615/IntJMultCompEng.2014010414 . hal01056157

\section{HAL Id: hal-01056157 \\ https://hal.science/hal-01056157}

Submitted on 11 Jun 2020

HAL is a multi-disciplinary open access archive for the deposit and dissemination of scientific research documents, whether they are published or not. The documents may come from teaching and research institutions in France or abroad, or from public or private research centers.
L'archive ouverte pluridisciplinaire $\mathbf{H A L}$, est destinée au dépôt et à la diffusion de documents scientifiques de niveau recherche, publiés ou non, émanant des établissements d'enseignement et de recherche français ou étrangers, des laboratoires publics ou privés. 


\title{
NONLOCAL/COARSE-GRAINING HOMOGENIZATION OF LINEAR ELASTIC MEDIA WITH NON-SEPARATED SCALES USING LEAST- SQUARE POLYNOMIAL FILTERS
}

\author{
Julien Yvonnet* \& Guy Bonnet \\ Université Paris-Est, Laboratoire Modélisation et simulation Multi Echelle, 5 Bd Descartes, \\ F-77454 Marne-la-Vallée Cedex 2, France
}

*Address all correspondence to Julien Yvonnet, E-mail: julien.yvonnet@univ-paris-est.fr

In this paper, a nonlocal computational method is proposed to construct a mesoscopic (coarse-grained) model of linear elastic heterogeneous materials in the case of nonseparated scales. The framework, introduced in our previous paper (Yvonnet and Bonnet, 2014), extends the classical homogenization framework by using low-pass filters operators instead of averaging operators, and Green's nonlocal functions instead of localization operators. In the present work, we introduce a filtering procedure based on least-square polynomial approximation to avoid the numerical drawbacks of Gaussian filters in finite domains. The complete associated homogenization scheme is described, as well as a numerical procedure based on finite elements to compute the different homogenized operators from a unit cell. The methodology is validated by analyzing both local and mesoscopic mechanical fields in structures where heterogeneities are of comparable size with respect to the loading characteristic fluctuation wavelength.

KEY WORDS: homogenization, nonseparated scales, nonlocal elasticity, coarse-graining, structures, multiscale methods

\section{INTRODUCTION}

Classical homogenization assumes separation of scales. This assumption is only valid when the characteristic fluctuation wavelength of an applied strain field over a given microstructure is supposed to be large compared with the scale of the microstructure. Otherwise, the scales are considered as nonseparated and the overall effective behavior at a given point depends on the strain states at other points in the neighborhood, defining a nonlocal effective behavior (Beran and McCoy, 1970). Such situations can occur when: (a) the heterogeneities composing the material of a structure have a characteristic length nearing the one of fluctuations of the applied load; (b) in the dynamic case, when elastic waves propagating through the material have a period not much larger than heterogeneities (Porubov et al., 2009); (c) for microstructures with complex morphologies which are continuously defined and imbricated through the scales, like in concrete (Giacco et al., 2009) or biomaterials. In this configuration, the details of heterogeneities become important at the scale of the structure, but a full description must be avoided unless yielding intractable computational times.

Defining an equivalent medium in the case of nonseparated scales is a quite old topic and finds its origin in homogenization at the atomistic scale (Kroner, 1967). A first category of methodology and theories is based on nonlocal elasticity theories. Usually, these methods use a nonlocal operator to define the behavior, which depends on a nonlocal definition of the strains. For example, the Eringen model (Eringen and Edelen, 1972) [see also Gao (1999) for a more recent asymmetric theory of nonlocal elasticity] defines the effective stress as the spatial convolution of the strain with a nonlocal elastic operator containing a monotonically decreasing non-negative function of the distance, typically described by Gauss-shaped or bell-shaped functions. These types of nonlocal elastic models are however 
empirical and not founded on a microstructural analysis. Furthermore, taking into account holes or cracks in such models constitutes a tough issue (Polizzotto, 2001).

A second class of approaches is based on gradient models. Higher-gradient continuum models can be derived as approximations of nonlocal models by means of truncated Taylor's series, see, e.g. Peerlings et al. (1996). Many gradient models and related techniques have been proposed, including among many others: (i) methodologies based on gradient of strain or higher derivatives of the strain (Mindlin, 1964; Mindlin and Eshel, 1968; Toupin, 1964); (ii) approaches based on couple-stress formulations [see Bouyge et al. (2001b); Ostoja-Starzewski et al. (1999); Toupin (1964); Willis (1983)]. In Kouznetsova et al. (2002), nonlinear computational schemes with two scales were proposed. See Bouyge et al. (2001a,b); Geers et al. (2007); Kouznetsova et al. (2002, 2004); Yuan and Tomita (2008) for other works deriving constitutive equations of generalized media through higher-order boundary conditions on the unit cell and use of generalized Hill-Mandel condition; (iii) Effective linear Cosserat medium (Forest et al., 1999; Forest and Sab, 1998), and (iv) methods based on series expansions (Boutin, 1996; Smyshlyaev and Cherednichenko, 2000; Tran et al., 2012). Note that some nonlocal constitutive equations, including stress-couple formulations have the drawback to maintain a gradient model even in the limit case of homogeneous microstructures (Yuan and Tomita, 2008).

New computational approaches using the features of nonlocal techniques have been proposed recently. In Kruch and Forest (1998), a simple relocalization procedure was introduced to compute local fields in the case of non-constant mesoscale fields, but inducing significant errors even in the displacement field of the structure for some microstructures and loading conditions, as shown in Forest (1998). In Fish et al. (2012), the effects of inelastic strains are replaced by nonlocal strains in a staggered algorithm to reduce computational complexity. In Coenen et al. (2012), a multiscale method was proposed to relate micro to macro damage in the case of nonseparated scales. In Hui and Oskay (2013), a nonlocal homogenization method was proposed to analyze wave dispersion and energy dissipation in biomaterials viscoelastic composites subjected to dynamic loading conditions. In Bacigalupo and Gambarotta (2011), elastic orthotropic moduli of a masonry microstructure were compared according to Cosserat, couple-stresses, and second-order homogenization and the validity of these models was discussed.

The purpose of the present work is to propose, in the continuation of Yvonnet and Bonnet (2014), a new nonlocal/ coarse-graining homogenization method, involving the following features:

i. Provide a fully microscopically based nonlocal homogenization framework, without any empirical assumptions;

ii. Define a generalization of classical homogenization, where mesoscopic models can be continuously constructed from the microscopic scale up to the macroscale, depending on the choice of the filter;

iii. Avoid issues of nonlocal elasticity when the microstructure contains holes or cracks;

iv. Being able to reproduce the effective strain and stress fields in a heterogeneous structure with large heterogeneities;

v. Operate in a simple, classical finite element framework.

To the best of the author's knowledge, such methodology has not been proposed so far.

The remainder of this manuscript is organized as follows. In Section 2, the problem of homogenization for nonseparated scales is formulated. A filtering process based on a least-square polynomial approximation is defined. Relations between mesoscopic stress and strain are formulated. Finally, the local problem and associated boundary conditions over the unit cell are defined. In Section 3, an associated numerical procedure based on finite elements is described. Finally, numerical examples are proposed in Section 4 to validate both homogenized models and re-localization processes.

\section{PROBLEM OF HOMOGENIZATION FOR NON-SEPARATED SCALES}

\subsection{Definition of Local and Mesoscopic Fields}

In Yvonnet and Bonnet (2014), we introduced a consistent nonlocal homogenization framework based on Gaussian filters for dealing with heterogeneous elastic media in the case of nonseparated scales. In the present paper, we propose 
to replace the Gaussian filters which were used previously to smooth the fine fluctuations fields, by a new linear filter operator which has strong numerical advantages. First, we define two scales, one scale associated with fine scale strain and stress fields $\varepsilon(\mathrm{x})$ and $\sigma(\mathrm{x})$, and another (upper) scale called "mesoscale," associated with strain and stress fields denoted by $\hat{\varepsilon}(\mathbf{x})$ and $\hat{\boldsymbol{\sigma}}(\mathbf{x})$, respectively, and called "mesoscopic" strain and stress fields. These fields are related to each other through the following relations:

$$
\begin{aligned}
\hat{\varepsilon}(\mathrm{x}) & =\mathcal{F}[\mathcal{\varepsilon}(\mathrm{x})], \\
\hat{\boldsymbol{\sigma}}(\mathrm{x}) & =\mathcal{F}[\boldsymbol{\sigma}(\mathrm{x})],
\end{aligned}
$$

where $\mathcal{F}($.$) is a linear operator, acting as a low-pass filter on the fine scale fluctuations. Associating \mathcal{F}$ with a characteristic length $h$ related to the fluctuations observation at the mesoscale, the following properties are assumed for $\mathcal{F}:$

$$
\begin{aligned}
\lim & \mathcal{F}_{h \rightarrow 0}^{h}[\varepsilon(x)]=\varepsilon(x), \\
\lim \underset{h \rightarrow \infty}{\mathcal{F}^{h}}[\varepsilon(x)] & =\langle\varepsilon(x)\rangle .
\end{aligned}
$$

An example of such operator is the Gaussian convolution product (Yvonnet and Bonnet, 2014). However, we have shown in the mentioned paper that in a numerical context, errors are induced due to some boundary effects in finite domains, associated with nonperiodic mesoscopic strain fields prescribed over the unit cell. In the present work, we propose a new filtering procedure based on a least-square polynomial fitting, and the associated nonlocal homogenization scheme, which alleviates these issues.

\subsection{Localization Problem}

Following Yvonnet and Bonnet (2014), we introduce the following split of microscopic strain into a filtered (mesoscopic) part and a remaining fluctuation $\tilde{\varepsilon}(\mathrm{x})$ :

$$
\varepsilon(\mathbf{x})=\hat{\varepsilon}(\mathbf{x})+\tilde{\varepsilon}(\mathbf{x})
$$

Let us consider a unit cell defined in a domain $\Omega \in \mathbb{R}^{D}, D$ being the dimension of the space, with boundary $\partial \Omega$. We define the following localization problem on the unit cell for nonseparated scales: assuming known an applied (nonconstant) mesoscopic strain field $\hat{\varepsilon}(\mathrm{x})$, find $\varepsilon(\mathrm{x})$ satisfying:

$$
\nabla \cdot[\boldsymbol{\sigma}(\mathrm{x})]=0 \text { in } \Omega
$$

and

$$
\mathcal{F}[\varepsilon(x)]=\hat{\varepsilon}(x) \text { in } \Omega,
$$

with

$$
\sigma(\mathrm{x})=\mathbb{C}(\mathrm{x}): \varepsilon(\mathrm{x})
$$

where $\mathbb{C}(\mathrm{x})$ is a fourth-order elasticity tensor. Condition (2.7) is an extension of the classical averaging condition of homogenization in the case of separated scales, as shown in the above reference. It can be interpreted as follows: the filtered part of the compatible strain field satisfying (2.6)-(2.8) must match $\hat{\varepsilon}(\mathrm{x}) \forall \mathrm{x} \in \Omega$.

\subsection{Nonlocal (Coarse-Graining) Mesoscopic Homogenization}

\subsubsection{Effective Constitutive Relationships}

Introducing (2.5) and (2.8) into (2.6) we obtain the new localization problem:

$$
\begin{gathered}
\nabla \cdot[\mathbb{C}(\mathrm{x}): \tilde{\varepsilon}(\mathrm{x})]=-\nabla \cdot[\mathbb{C}(\mathrm{x}): \hat{\varepsilon}(\mathrm{x})] \text { in } \Omega, \\
\mathcal{F}(\tilde{\varepsilon}(\mathrm{x}))=0 \text { in } \Omega .
\end{gathered}
$$


Condition (2.10) cannot be simply formulated as a boundary condition. Its enforcement is defined in Section 2.5. Assuming a unique solution for problem (2.9)-(2.10) thanks to some appropriate boundary conditions (defined in Section 2.5$), \tilde{\varepsilon}(\mathrm{x})$ can be related to $\hat{\varepsilon}(\mathrm{x})$ through an appropriate Green's function tensor $\hat{\Gamma}$ as

$$
\tilde{\varepsilon}(\mathbf{x})=-\int_{\Omega} \hat{\Gamma}(\mathbf{x}, \mathbf{y}): \hat{\varepsilon}(\mathbf{y}) d \mathbf{y}
$$

where $d \mathbf{y}$ indicates integration with respect to the $\mathrm{y}$ space variable. The definition of $\hat{\Gamma}(\mathrm{x}, \mathrm{y})$ can be found in Yvonnet and Bonnet (2014). Using (2.5) we obtain the expression of the microscopic strain field as a function of the mesoscopic one by

$$
\varepsilon(\mathbf{x})=\int_{\Omega} \hat{\mathbb{A}}(\mathbf{x}, \mathbf{y}): \hat{\varepsilon}(\mathbf{y}) d \mathbf{y},
$$

with $\hat{\mathbb{A}}(\mathrm{x}, \mathrm{y})=\mathbb{I} \delta(\mathrm{x}-\mathrm{y})-\hat{\Gamma}(\mathrm{x}, \mathrm{y}), \mathbb{I}$ being the fourth-order identity tensor. Using (2.12), (2.8), and (2.2) we can close the relation between mesoscopic stress and strain fields as

$$
\hat{\boldsymbol{\sigma}}(\mathbf{x})=\int_{\Omega} \hat{\mathbb{C}}(\mathbf{x}, \mathbf{y}): \hat{\varepsilon}(\mathbf{y}) d \mathbf{y}
$$

where

$$
\hat{\mathbb{C}}(\mathrm{x}, \mathrm{y})=\mathcal{F}\{\mathbb{C}(\mathrm{x}): \hat{\mathbb{A}}(\mathrm{x}, \mathrm{y})\}
$$

is a nonlocal elastic tensor defined in $\Omega$. Note that the constitutive law (2.13)-(2.14) is general for linear filter operators satisfying conditions (2.3)-(2.4), and then remains valid when $\mathcal{F}$ is chosen as a Gaussian filter. Another remark is that in contrast to classical nonlocal elasticity (Eringen and Edelen, 1972), the present nonlocal elasticity operator has not translational invariance, i.e., $\hat{\mathbb{C}}(\mathrm{x}, \mathrm{y}) \neq \hat{\mathbb{C}}(\mathrm{x}-\mathrm{y})$. This constitutes a key feature of the the model to continuously define mesoscopic constitutive laws from the microscopic scale up to the macroscale.

\subsubsection{Limit Cases}

- Separated scales: $h / \alpha \gg 1$; when the scales are separated, i.e., when the characteristic length $h$ related to the fluctuations at the mesoscopic scale is much larger than the characteristic length fluctuation of the microscopic scale $\alpha$, we have shown in Yvonnet and Bonnet (2014) that due to property (2.4), the constitutive relationship (2.13) degenerates into the classical homogenized constitutive law for elastic materials:

$$
\hat{\sigma}=\langle\mathbb{C}(\mathrm{x}): \mathbb{A}(\mathrm{x})\rangle: \hat{\varepsilon},
$$

with $\mathbb{A}(\mathrm{x})$ the classical localization tensor such that $\varepsilon(\mathrm{x})=\mathbb{A}(\mathrm{x}): \hat{\varepsilon}$. In that case, the mesoscopic fields match the macroscopic fields, $\hat{\sigma}=\langle\sigma(x)\rangle=\bar{\sigma}, \hat{\varepsilon}=\langle\varepsilon(x)\rangle=\bar{\varepsilon}$.

- Microscopic scale: $h / \alpha \simeq 1$; in the other limit case, when $h \simeq \alpha$, we show that we recover the local constitutive relationships. For $h \simeq \alpha$, we have

$$
\lim _{h \rightarrow 0} \mathcal{F}_{h \rightarrow}^{h}(\varepsilon(x)) \simeq \varepsilon(x)
$$

Then, condition (2.10) gives

$$
\mathcal{F}[\tilde{\varepsilon}(\mathrm{x})] \simeq \tilde{\varepsilon}(\mathrm{x})=0 \text { in } \Omega
$$

and (2.5) yields

From (2.12), we obtain

$$
\varepsilon(\mathrm{x}) \simeq \hat{\varepsilon}(\mathrm{x})
$$

$$
\hat{\mathbb{A}}(\mathrm{x}, \mathrm{y}) \simeq \mathbb{I} \delta(\mathrm{x}-\mathrm{y}) .
$$

Finally, Eq. (2.14) degenerates into

$$
\hat{\mathbb{C}}(\mathrm{x}, \mathrm{y}) \simeq \mathcal{F}[\mathbb{C}(\mathrm{x}): \mathbb{I} \delta(\mathrm{x}-\mathrm{y})] \simeq \mathbb{C}(\mathrm{x}): \mathbb{I} \delta(\mathrm{x}-\mathrm{y}) .
$$

Introducing (2.20) in (2.13) we recover the local microscopic constitutive law (2.8). 
- Homogeneous material; when the volume fraction of inclusions in the microstructure tends to zero, we have shown in Yvonnet and Bonnet (2014) that we recover a local elastic constitutive law.

In conclusion, for the extreme cases $h / \alpha \gg 1$ and $h / \alpha \simeq 1$, we recover the classical homogenization scheme for separated scales and the local microscopic constitutive law, respectively. The present theory then allows constructing a range of homogenized models between these two extreme cases in a continuous and consistent way by varying $h$, or in the discrete framework presented below, by varying the coarse mesh discretization.

\subsection{Filter Operator Based on a Least-Square Polynomial Projection}

We define the following filter process, which consists of a least-square projection of the microscopic strain field over a piece-wise polynomial basis, whose characteristic length $h$ is associated with the mesoscopic characteristic length of fluctuation of fields. This length is assumed to be given, and can be associated with the characteristic wavelength of the applied load. We define

$$
\mathcal{F}\left[\varepsilon_{i j}(\mathbf{x})\right]=\sum_{p=1}^{P} M^{p}(\mathbf{x}) \hat{\varepsilon}_{i j}^{p},
$$

where $M^{p}(\mathrm{x})$ are piece-wise polynomial basis functions (e.g., finite element shape functions) and $\hat{\varepsilon}_{i j}^{p}$ are coefficients, interpreted as the nodal values of the mesoscopic strain field at some nodes of a coarse grid associated with the mesoscopic mesh [see Fig. 1(a)], for each component $i j$. These values are assumed associated with a compatible mesoscopic strain field.

Given the fine scale strain field $\varepsilon(\mathrm{x})$ on a discrete fine mesh composed of $N$ nodes $\mathrm{x}^{m}, m=1, \ldots, N$, the unknown coefficients $\hat{\varepsilon}_{i j}^{p}$ are required to minimize the distance between the approximation and the given fine scale strain field in the least square sense. Let us define $U$ such that

$$
U=\sum_{m=1}^{N}\left(\sum_{p=1}^{P} M^{p}\left(\mathrm{x}^{m}\right) \hat{\varepsilon}_{i j}^{p}-\varepsilon_{i j}\left(\mathrm{x}^{m}\right)\right)^{2} .
$$

Optimality conditions give

$$
\frac{d U}{d \hat{\varepsilon}_{i j}^{q}}=0, q=1,2, \ldots, N
$$

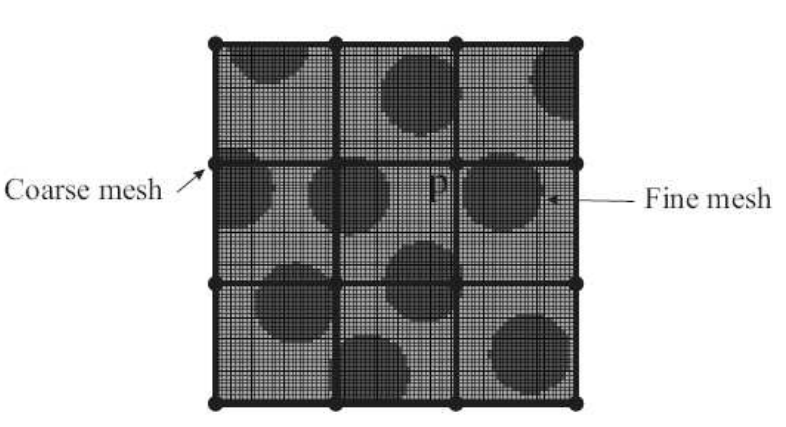

(a)

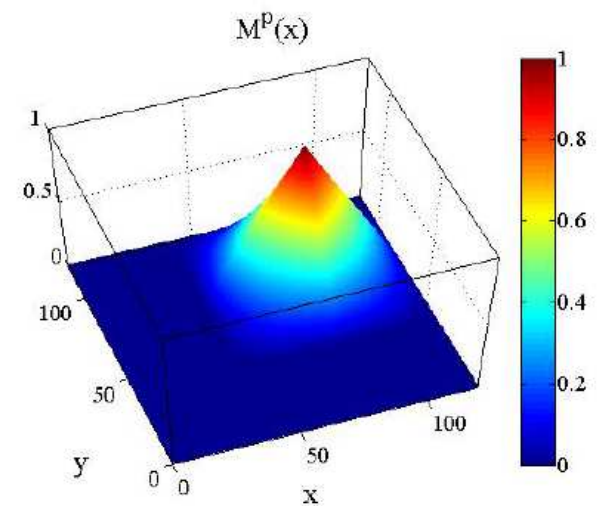

(b)

FIG. 1: (a) Fine grid used to approximate the microscopic strain field $\varepsilon(\mathrm{x})$ and coarse grid used to approximate the mesoscopic strain field $\hat{\varepsilon}(\mathrm{x})$; (b) shape function $M^{p}(\mathrm{x})$ of node $p$ on the coarse grid. 
leading to

$$
2 \sum_{m=1}^{N} M^{q}\left(\mathrm{x}^{m}\right)\left[\sum_{p=1}^{P} M^{p}\left(\mathrm{x}^{m}\right) \hat{\varepsilon}_{i j}^{p}-\varepsilon_{i j}\left(\mathrm{x}^{m}\right)\right]=0, q=1,2, \ldots, N,
$$

or

$$
\sum_{p=1}^{P}\left(\sum_{m=1}^{N} M^{p}\left(\mathrm{x}^{m}\right) M^{q}\left(\mathbf{x}^{m}\right)\right) \hat{\varepsilon}_{i j}^{p}=\sum_{m} M^{q}\left(\mathbf{x}^{m}\right) \varepsilon_{i j}\left(\mathbf{x}^{m}\right) .
$$

Then the coefficients $\hat{\varepsilon}_{i j}^{p}, p=1,2, \ldots, N$ are found by solving the following system:

$$
\mathrm{Au}=\mathrm{b},
$$

where

$$
A_{p q}=\sum_{m=1}^{N} M^{p}\left(\mathrm{x}^{m}\right) M^{q}\left(\mathrm{x}^{m}\right), \quad b_{q}=\sum_{m=1}^{N} M^{q}\left(\mathrm{x}^{m}\right) \varepsilon_{i j}\left(\mathrm{x}^{m}\right),
$$

and where $\mathbf{u}=\left[\hat{\varepsilon}_{i j}^{1}, \hat{\varepsilon}_{i j}^{2}, \ldots, \hat{\varepsilon}_{i j}^{N}\right]$. It is noteworthy that Eq. (2.26) implies the filtering relation (2.10). Indeed, it is clear that the mesoscopic strain field is invariant through the filtration operator, $U$ being null when introducing $\mathcal{F}(\hat{\varepsilon})$ into the filtration process. This induces that $\mathcal{F}[\varepsilon(\mathrm{x})]=\mathcal{F}[\hat{\varepsilon}(\mathrm{x})]$, which leads to (2.10).

Examples of filtered strain fields using this process are shown in Figs. 2 and 3, when a basis of linear and bilinear piece-wise (FEM) functions are employed in 1D and 2D, respectively. In this work, we use bilinear basis functions for $M^{p}(\mathrm{x})$, but other functions with higher continuity (quadratic, splines) can be used without additional costs.

This procedure has the advantage to alleviate the boundary effects encountered for filtered nonperiodic fields over a finite domain $\Omega$. In order to show this advantage of the new numerical procedure, we compare in Fig. 2(b) the filtered strain obtained from applying a Gaussian filter (convolution operator) on an oscillating field with a filtered part being linear. To evaluate the field outside the domain, the linear field is repeated periodically. We can clearly observe that the piece-wise polynomial filter operator removes the spurious boundary effects in the finite domain. In addition, an obvious advantage of this new homogenization scheme is to use a filter which is related to the meshing at the mesoscopic scale.

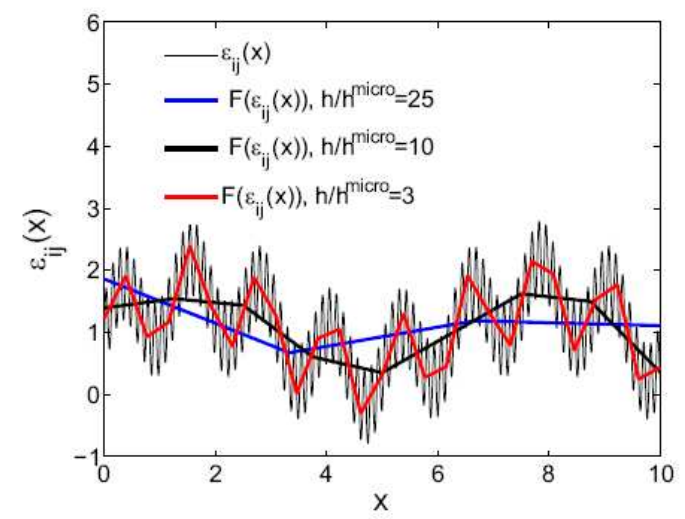

(a)

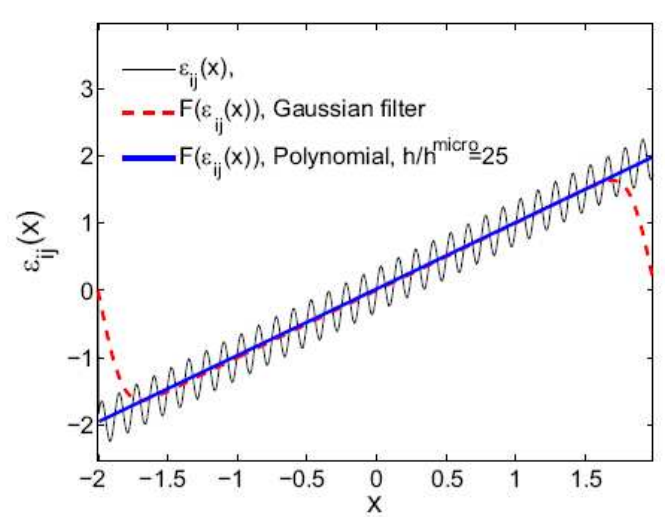

(b)

FIG. 2: (a) Illustration of the least-square polynomial filter for different values of $h$; (b) Illustration of spurious effects induced by a Gaussian filter for a non-periodic fluctuation field in a finite domain. 


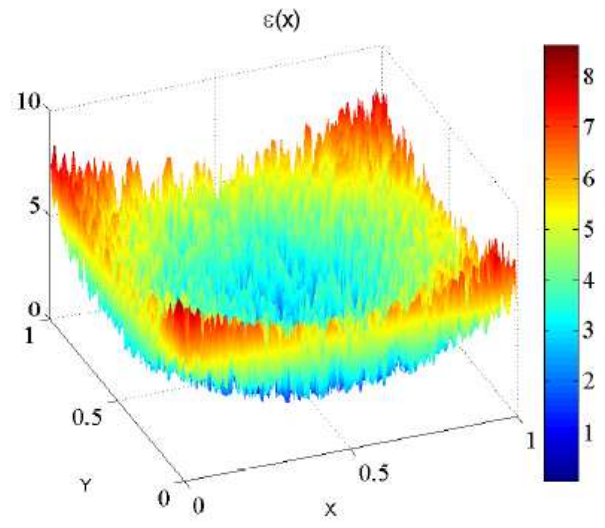

(a)

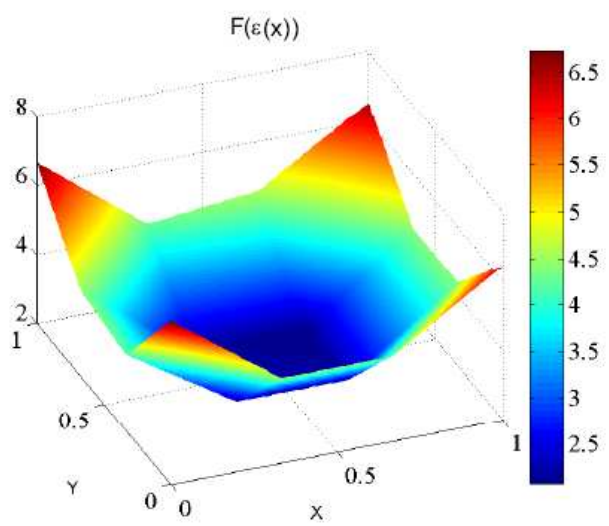

(b)

FIG. 3: Illustration of the action of the least-square filter on an oscillating strain field in 2D: (a) strain field; (b) filtered strain field by least-square polynomial fit with $h=1 / 3$.

\subsection{Boundary Conditions and Solving Procedure for the Localization Problem}

To enforce the nontrivial condition (2.7), we propose the following procedure: we define an auxiliary strain field $\mathbf{e}(\mathbf{x})$ defined by

$$
\tilde{\varepsilon}(\mathrm{x})=\mathbf{e}(\mathrm{x})-\mathcal{F}[\mathbf{e}(\mathrm{x})] .
$$

Invoking the following property of the least-square polynomial filter operator:

$$
\mathcal{F}(\mathcal{F}(\mathbf{e}(\mathrm{x})))=\mathcal{F}[\mathrm{e}(\mathrm{x})],
$$

and introducing (2.28) in (2.10) we can verify that due to the linearity of $\mathcal{F}$ we have

$$
\mathcal{F}[\tilde{\varepsilon}(\mathrm{x})]=\mathcal{F}(\mathbf{e}(\mathrm{x})-\mathcal{F}(\mathrm{e}(\mathrm{x})))=\mathcal{F}(\mathbf{e}(\mathrm{x}))-\mathcal{F}(\mathcal{F}[\mathrm{e}(\mathrm{x})])=0 .
$$

Then, using definition (2.28) and (2.9) the new localization problem is defined by seeking $\mathrm{e}(\mathrm{x})$ satisfying

$$
\nabla \cdot(\mathbb{C}(\mathrm{x}):[\mathrm{e}(\mathrm{x})-\mathcal{F}(\mathrm{e}(\mathrm{x}))])=-\nabla \cdot(\mathbb{C}(\mathrm{x}): \hat{\varepsilon}(\mathrm{x})) \text { in } \Omega .
$$

Condition (2.10) implies that

$$
\langle\tilde{\varepsilon}(\mathrm{x})\rangle=0,
$$

which is satisfied for any value of the spatial average $\langle\mathbf{e}(\mathrm{x})\rangle$. Obviously, this spatial average can be fixed at any value. So, we choose

$$
\langle\mathrm{e}(\mathrm{x})\rangle=0 .
$$

Condition (2.33) is classically verified for the two possible sets of boundary conditions:

$$
\mathbf{u}^{e}(\mathbf{x})=0 \text { on } \partial \Omega,
$$

or

$$
\mathbf{u}^{e}(\mathbf{x}) \text { periodic on } \Omega,
$$

where $\mathbf{u}^{e}$ is a compatible displacement field such that $\mathbf{e}(\mathbf{x})=\varepsilon\left(\mathbf{u}^{e}(\mathbf{x})\right)$, with $\varepsilon()=.1 / 2\left[\nabla()+.\nabla^{T}().\right]$. To summarize, the new problem is defined by Eq. (2.31) with boundary conditions (2.34) or (2.35). In the present work, 
we have adopted the second set of boundary conditions. The presence of the nonlocal operator in the left-hand term of (2.31) induces a numerical difficulty, as the stiffness matrix associated with this linear operator is fully populated. Here we alleviate this difficulty by defining the following iterative scheme: starting from an initialized solution $\mathrm{e}^{0}(\mathrm{x})$, e.g., $\mathbf{e}^{0}(\mathbf{x})=0$, we seek at each iteration $n$ of the following scheme the sought field $\mathbf{e}^{n+1}(\mathbf{x})$ such that

$$
\nabla \cdot\left(\mathbb{C}(\mathrm{x}): \mathrm{e}^{n+1}(\mathrm{x})\right)=-\nabla \cdot\left(\mathbb{C}(\mathrm{x}): \mathcal{F}\left(\mathbf{e}^{n}(\mathrm{x})\right)\right)+\nabla \cdot[\mathbb{C}(\mathrm{x}): \hat{\varepsilon}(\mathrm{x})]
$$

until a convergence criterion is reached. At convergence, the strain field is recovered by:

$$
\mathcal{E}(\mathrm{x}) \simeq \hat{\varepsilon}(\mathrm{x})+\mathrm{e}^{n+1}(\mathrm{x})-\mathcal{F}\left[\mathrm{e}^{n+1}(\mathrm{x})\right] .
$$

The convergence of this scheme has been demonstrated in the case of Gaussian filters in Yvonnet and Bonnet (2014) for any starting strain field. In the present case, the convergence of this process is ensured a priori only if the spectral radius of the linear operator associated with the recurrence process is lower than 1 . In our numerical experiences, the recurrence process did converge for all studied cases, and about 20 iterations were necessary to reach an error of $5 \times 10^{-2}$. It seems therefore that the result obtained for Gaussian filters is also valid for the least-square filtering process. However, a more careful study of this convergence process is postponed to future work.

\section{COMPUTATIONAL PROCEDURE}

In this section, we describe a discrete formulation associated with the continuous framework developed in Sections 2.3 and 2.5, and an associated FEM computational procedure to construct the localization operators for the mesoscale, from computations on the unit cell $\Omega$. Note that other numerical methods such as XFEM (Moës et al., 2003), FFT (Moulinec and Suquet, 1994), or related methods, (Yvonnet, 2012) could be alternatively considered for solving the local problem. Even though similar features seemed shared with other techniques involving fine scale and coarse scale coupling [see, e.g., Duarte and Kim (2008)], where fine scale solutions are used to enrich the kinematics of the coarse scale within the same model for both scale, here the coarse scale model is straightforwardly built from the fine scale model by a homogenization process. The proposed technique is also different from multigrid techniques, where solutions from coarse meshes are used to accelerate the computation of a problem on a finer grid in a context of iterative solvers.

\subsection{Discrete Formulation}

Following Yvonnet and Bonnet (2014), we define $\hat{\varepsilon}(\mathrm{x})$ in the form

$$
\hat{\varepsilon}_{i j}(\mathrm{x}) \simeq \sum_{p} M^{p}(\mathrm{x}) \hat{\varepsilon}_{i j}^{p},
$$

where the nodal values $\hat{\varepsilon}_{i j}^{p}$ are given and as previously, $M^{p}$ are interpolations functions. Using the filter operator $\mathcal{F}$ defined by (2.21), we obtain the following relationships:

$$
\hat{\boldsymbol{\sigma}}(\mathbf{x})=\sum_{p} \hat{\mathbb{C}}^{p}(\mathbf{x}): \hat{\varepsilon}^{p}
$$

with

$$
\hat{\mathbb{C}}^{p}(\mathrm{x})=\mathcal{F}\left\{\mathbb{C}(\mathrm{x}): \hat{\mathbb{A}}^{p}(\mathrm{x})\right\}
$$

and

$$
\widehat{\mathbb{A}}^{p}(\mathrm{x})=M^{p}(\mathrm{x}) \mathbb{I}+\Psi^{p}(\mathrm{x})-\mathcal{F}\left(\Psi^{p}(\mathrm{x})\right),
$$

where $\Psi^{p}(\mathrm{x})$ are transformation tensors associated with each node $p$ of the coarse mesh [see Fig. 4(b)], defined by

$$
\Psi_{i j k l}^{p}(\mathbf{x})=e_{i j}^{p,(k l)}(\mathbf{x})
$$


where $\mathrm{e}^{p,(k l)}$ is the strain solution field obtained by solving on the unit cell the elementary problem:

$$
\nabla \cdot\left(\mathbb{C}(\mathrm{x}):\left[\mathrm{e}^{p,(k l)}(\mathrm{x})-\mathcal{F}\left\{\mathrm{e}^{p,(k l)}(\mathrm{x})\right\}\right]\right)=-\nabla \cdot\left(\mathbb{C}(\mathrm{x}): \chi^{p,(k l)}(\mathrm{x})\right)
$$

with boundary conditions (2.34) or (2.35) and where $\chi^{p,(k l)}(\mathrm{x})$ is a nonuniform eigenstrain prescribed over the support of $M^{p}(\mathrm{x})$ and defined as

$$
\chi^{p,(k l)}(\mathbf{x})=\frac{M^{p}(\mathbf{x})}{2}\left(\mathbf{v}_{k} \otimes \mathbf{v}_{l}+\mathbf{v}_{l} \otimes \mathbf{v}_{k}\right),
$$

where $\mathbf{v}_{i}$ are the base vectors of a Cartesian coordinate system.

Remark It can be seen that the strain fields defined by (3.1) are not all compatible. However, the method intends to produce the microscopic response to a given mesoscopic strain field obtained by a FEM solution of the response of the structure. This mesoscopic strain field must therefore be compatible, being the solution of a FEM modeling.

\subsection{Computation of Transformation Tensors with FEM}

Setting $\mathrm{e}^{p,(k l)}(\mathrm{x})=\beta(\mathrm{x})$ for the sake of simplification of notations, the problem (3.6) is computed iteratively by solving the problem

$$
\nabla \cdot\left(\mathbb{C}(\mathrm{x}): \beta^{n+1}(\mathrm{x})\right)=-\nabla \cdot\left(\mathbb{C}(\mathrm{x}): \chi^{p,(k l)}(\mathrm{x})\right)+\nabla \cdot\left(\mathbb{C}(\mathrm{x}): \mathcal{F}\left(\beta^{n}(\mathrm{x})\right)\right),
$$

with either zero or periodic boundary conditions on the displacements $\mathbf{u}^{\beta}$ compatible with the strain field $\beta(x)$, i.e., $\varepsilon\left(\mathbf{u}^{\beta}(\mathrm{x})\right)=\beta(\mathrm{x})$. The weak form corresponding to (3.8) is given as follows: find $\mathbf{u}^{\beta}(\mathbf{x}) \in H^{1}(\Omega)$ and satisfying boundary conditions (2.35), such that

$$
\begin{gathered}
\int_{\Omega} \mathcal{\varepsilon}\left(\left[\mathbf{u}^{\beta}\right]^{n+1}(\mathbf{x})\right): \mathbb{C}(\mathbf{x}): \mathcal{\varepsilon}[\delta \mathbf{u}(\mathbf{x})] d \Omega=-\int_{\Omega} x^{p,(k l)}(\mathbf{x}): \mathbb{C}(\mathbf{x}): \varepsilon[\delta \mathbf{u}(\mathbf{x})] d \Omega \\
+\int_{\Omega} \mathcal{F}\left\{\varepsilon\left(\left[\mathbf{u}^{\beta}\right]^{n}(\mathbf{x})\right)\right\}: \mathbb{C}(\mathbf{x}): \mathcal{\varepsilon}(\delta \mathbf{u}(\mathbf{x})) d \Omega \quad \forall \delta \mathbf{u} \in H_{0}^{1}(\Omega),
\end{gathered}
$$

where $H_{0}^{1}(\Omega)$ is the usual Sobolev space containing functions defined on the unit cell. Using a usual FEM discretization for the fine mesh defined over $\Omega$, we obtain the linear system

$$
\mathbf{K} \mathbf{u}^{n+1}=\mathbf{f}^{p, k l}+\mathbf{f}^{n}
$$

where $\mathbf{K}$ is the classical FEM rigidity matrix

$$
\mathbf{K}=\int_{\Omega} \mathbf{B}^{T}(\mathbf{x}) \mathbf{C}(\mathbf{x}) \mathbf{B}(\mathbf{x}) d \Omega,
$$

and $\mathrm{f}$ is a body force vector due to the prescribed nonuniform eigenstrains

$$
\mathbf{f}^{p, k l}=\int_{\Omega} \mathbf{B}^{T}(\mathbf{x}) \mathbf{C}(\mathbf{x}) \mathbf{X}^{p, k l}(\mathbf{x}) d \Omega,
$$

where $\mathbf{B}$ is the matrix of shape function derivatives and $\mathbf{C}$ is the matrix form corresponding to the fourth-order tensor $\mathbb{C}$ and

$$
\mathbf{f}^{n}=\int_{\Omega} \mathbf{B}^{T}(\mathbf{x}) \mathbf{C}(\mathbf{x}) \varepsilon^{R}(\mathbf{x}) d \Omega .
$$


In the above, $\mathbf{X}^{p, k l}$ is the vector form of the second-order tensor $\chi^{p,(k l)}$. For example, in $2 \mathrm{D}$, there are three elementary eigenstrain tensors for each node $p$ :

$$
\begin{gathered}
\chi^{p, 11}(\mathrm{x})=M^{p}(\mathrm{x})\left[\begin{array}{ccc}
1 & 0 & 0 \\
0 & 0 & 0 \\
0 & 0 & 0
\end{array}\right] ; \chi^{p, 22}(\mathrm{x})=M^{p}(\mathrm{x})\left[\begin{array}{ccc}
0 & 0 & 0 \\
0 & 1 & 0 \\
0 & 0 & 0
\end{array}\right] \\
\chi^{p, 12}(\mathrm{x})=M^{p}(\mathrm{x})\left[\begin{array}{ccc}
0 & 1 / 2 & 0 \\
1 / 2 & 0 & 0 \\
0 & 0 & 0
\end{array}\right]
\end{gathered}
$$

The corresponding vectors $\mathbf{X}^{p, k l}$ are given by

$$
\begin{gathered}
\mathbf{X}^{p, 11}(\mathrm{x})=M^{p}(\mathrm{x})\left[\begin{array}{l}
1 \\
0 \\
0
\end{array}\right] ; \mathbf{X}^{p, 22}(\mathrm{x})=M^{p}(\mathrm{x})\left[\begin{array}{l}
0 \\
1 \\
0
\end{array}\right] ; \\
\mathbf{X}^{p, 12}(\mathrm{x})=M^{p}(\mathrm{x})\left[\begin{array}{l}
0 \\
0 \\
1
\end{array}\right] .
\end{gathered}
$$

Finally, $\varepsilon^{R}(\mathrm{x})$ is the vector form of the filtered strain field $\mathcal{F}\left\{\varepsilon\left(\left[\mathbf{u}^{\beta}\right]^{n}(\mathrm{x})\right)\right\}$ defined by applying the procedure described in Section 2.4 for each component of $\varepsilon\left(\left[\mathbf{u}^{\beta}\right]^{n}(\mathrm{x})\right)$.

In $2 \mathrm{D}, P \times 3$ problems must be solved on the fine mesh defined over the unit cell, and $P \times 6$ in $3 \mathrm{D}$, where $P$ is the number of nodes on the coarse mesh. Note that all these problems (including all the iterations of the iterative procedure) have the same matrix $\mathbf{K}$, which only needs to be assembled and decomposed once.

The overall algorithm for the preliminary computations is summarized as follows:

For each point $p$ of the coarse grid (see Fig. 4):

i. Solve the problem (3.6) with boundary conditions (2.35) for each set of indices $\{k, l\}$ until convergence is reached.

ii. Compute $\Psi^{p}(\mathrm{x})$ using (3.5).

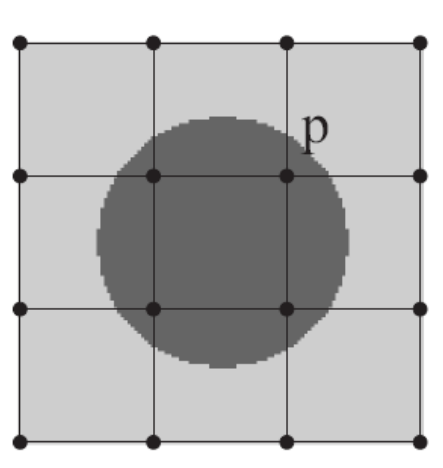

(a)

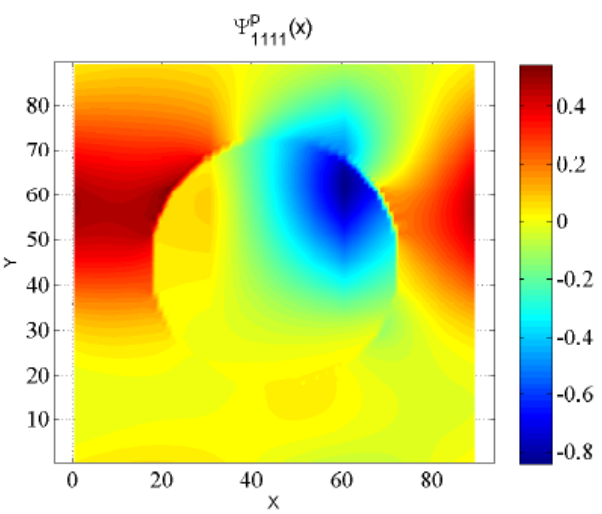

(b)

FIG. 4: (a) Coarse mesh and fine meshes covering the unit cell; (b) $\varepsilon_{11}(\mathrm{x})$-strain field obtained by prescribing a non-uniform eigenstrain $\chi^{p, 11}(\mathrm{x})=M^{p}(\mathrm{x}) \mathrm{v}_{1} \otimes \mathrm{v}_{1}$, and identified as $\Psi_{1111}^{p}(\mathrm{x})$. 
iii. Compute $\widehat{\mathbb{A}}^{p}(\mathrm{x})$ using (3.4) and store it.

iv. Compute $\hat{\mathbb{C}}^{p}(\mathrm{x})$ using (3.3) and store it. Only nodal values of $\hat{\mathbb{C}}^{p}(\mathrm{x})$ at the nodes of the coarse grid need to be stored. The full spatial description in the unit cell can be recovered through $\hat{\mathbb{C}}^{p}(\mathrm{x})=\sum_{i} M^{i}(\mathrm{x})\left[\hat{\mathbb{C}}^{p}\right]_{i}$, where $\left[\hat{\mathbb{C}}^{p}\right]_{i}$ are the nodal values of $\hat{\mathbb{C}}^{p}(\mathrm{x})$ on the coarse grid.

Once these tensors are computed and stored, for an arbitrary distribution of nodal values of the mesoscopic strain field $\hat{\varepsilon}^{p}$ over the coarse grid, we can compute:

i. The mesoscopic stress field $\hat{\boldsymbol{\sigma}}(\mathrm{x})$ using (3.2).

ii. The reconstructed local strain field through

$$
\varepsilon(\mathbf{x})=\sum_{p} \hat{\mathbb{A}}^{p}(\mathbf{x}) \hat{\varepsilon}^{p}
$$

iii. The reconstructed local stress field through

$$
\sigma(\mathrm{x})=\sum_{p} \mathbb{C}(\mathrm{x}): \hat{\mathbb{A}}^{p}(\mathrm{x}) \hat{\varepsilon}^{p} .
$$

\section{NUMERICAL EXAMPLES}

\subsection{Heterogeneous Structure}

In this first example, a heterogeneous beam structure is considered. Its geometry is depicted in Fig. 5(a).

The dimensions of the beams are $L=270 \mathrm{~mm}, H=90 \mathrm{~mm}$. Three holes with radii $R=H \sqrt{f / \pi}$, with $f$ $=0.3$ being the volume fraction, are positioned along the beam. The coordinates of the centers of holes are given by: $\{H / 2 ; H / 2\},\{3 H / 2 ; H / 2\}$, and $\{5 H / 2 ; H / 2\}$. Holes are modeled by an elastic material with very compliant properties, taken as $\lambda_{i n c}=10^{-3}=\mu_{i n c}=10^{-3} \mathrm{GPa}$, while the properties of the matrix are chosen as $\lambda_{\text {mat }}=$ $1=\mu_{m a t}=1 \mathrm{GPa}, \lambda$ and $\mu$ denoting the Lamé's elastic parameters. Introducing voids without its internal meshing would actually introduce a difficulty, related to applying the filter operator described in Section 2.4: in that case, the minimization problem is not defined over the whole cell domain and an extension of the filter definition would be required.

Plane strain is assumed. Obviously, a classical homogenized model would not be pertinent here, as the scales are clearly not separated. Furthermore, this test constitutes a difficult problem for other classical nonlocal models such as the Eringen model (Eringen and Edelen, 1972), which are known to fail in presence of holes or cracks in the domain (Polizzotto, 2001).

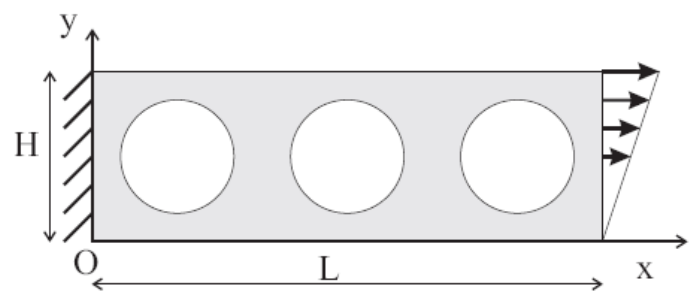

(a)

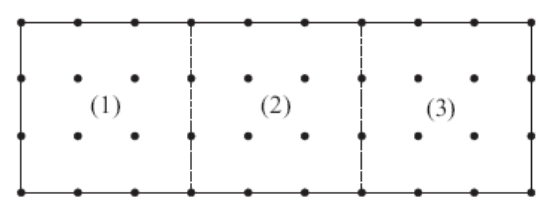

(b)

FIG. 5: (a) Geometry of the beam with structural holes subjected to bending; (b) coarse grid used to compute the mesoscopic solution. 
The beam is subjected to Dirichlet boundary conditions corresponding to a bending of the beam in the form

$$
\mathbf{u}(\mathbf{x})=\left\{\begin{array}{l}
u_{x}=\frac{x^{2} y}{\alpha} \\
u_{y}=-\frac{x^{3}}{3 \alpha}
\end{array} \quad \text { on } \partial W_{u}\right.
$$

where $\partial W_{u}$ is the boundary of the structure domain $W$, and $\alpha=1000$. The mesh is composed of regular 4-nodes bilinear elements and $270 \times 90$ nodes. A $10 \times 4$ coarse grid is associated with the structure for defining the mesoscopic model [see Fig. 5(b)].

The solution of this problem serves as a reference solution. The local strain and stress reference solutions, denoted by $\varepsilon_{i j}^{r e f}(\mathrm{x})$ and $\sigma_{i j}^{r e f}(\mathrm{x})$, correspond to the solution in the fully meshed structure. A mesoscopic reference solution, leading to $\mathcal{F}\left(\varepsilon_{i j}^{r e f}(\mathrm{x})\right)$ and $\mathcal{F}\left(\sigma_{i j}^{r e f}(\mathrm{x})\right)$, is obtained by applying the least-square polynomial fitting described in Section 2.4 on the coarse grid of Fig. 5(b).

To validate the present homogenization scheme, we use a single periodic cell from which localization and mesoscopic constitutive tensors $\widehat{\mathbb{A}}^{p}(\mathrm{x})$ and $\hat{\mathbb{C}}(\mathrm{x})$ are precomputed. The geometry of the unit cell is depicted in Fig. 4(a): it is constituted by a unit square containing a centered hole with volume fraction $f=0.3$. The microscopic mesh is composed of $90 \times 90$ regular 4-node bilinear elements. The material properties of the hole (modeled as a soft inclusion) and of the matrix are the same as for the structure problem. A coarse $4 \times 4$ grid is associated with the unit cell domain, which allows to obtain the tensors $\hat{\mathbb{A}}^{p}(\mathrm{x})$ and $\hat{\mathbb{C}}(\mathrm{x})$ associated with each point of the coarse grid in the unit cell, following the procedure described in Section 3.2. Then, we apply the corresponding reference mesoscopic strain field solution $\hat{\varepsilon}_{i j}(\mathrm{x})=\mathcal{F}\left(\varepsilon_{i j}^{r e f}(\mathrm{x})\right)$ of all subdomains (1)-(3) in Fig. 5(b) at control nodes of the $4 \times 4$ coarse grid on the unit cell. The reconstructed strain and stress fields computed by (3.18) and (3.19) are denoted by $\varepsilon_{i j}(\mathrm{x})$ and $\sigma_{i j}(\mathrm{x})$. We then compute the mesoscopic strain by computing $\mathcal{F}\left(\varepsilon_{i j}(\mathrm{x})\right)$ and the mesoscopic stress by (3.2).

Results are provided in Figs. 6-9, showing a good agreement of the reconstructed local and mesoscopic fields with respect to the reference solution. We can appreciate that the reconstructed mesoscopic strain field is exactly reproduced with respect to the reference solution, demonstrating that condition (2.7) is verified. Note that in this work, the structure problem using the mesoscopic model was not solved on the coarse grid. Indeed, an associated non standard finite element procedure is necessary for solving the mesoscopic model. Such a procedure will be defined in a forthcoming paper.

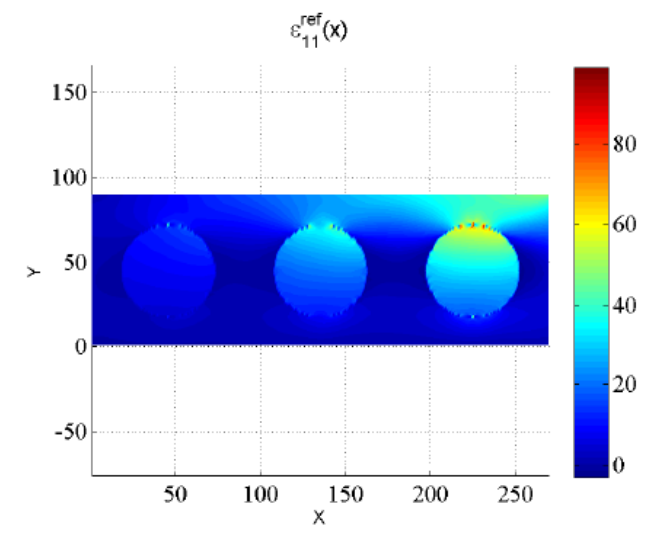

(a)

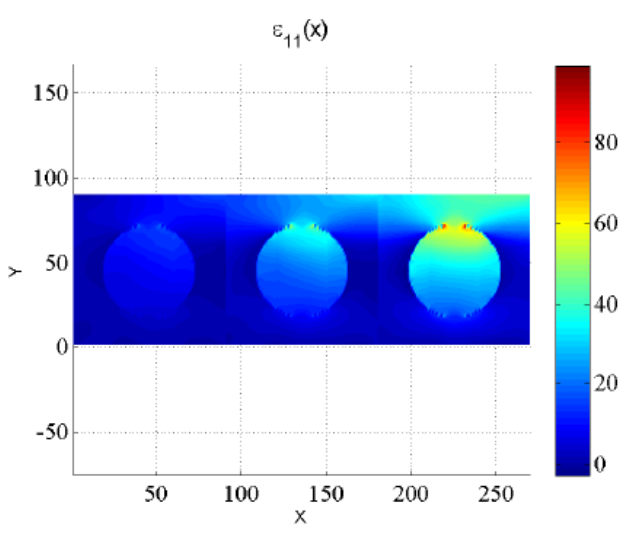

(b)

FIG. 6: (a) Reference solution for local strain field $\varepsilon_{11}^{r e f}(\mathrm{x})$; (b) Reconstructed solution from the coarse-grained model. 


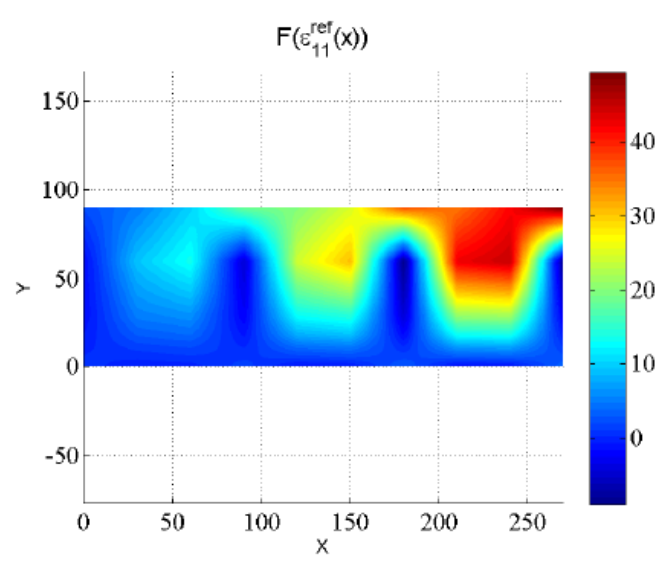

(a)

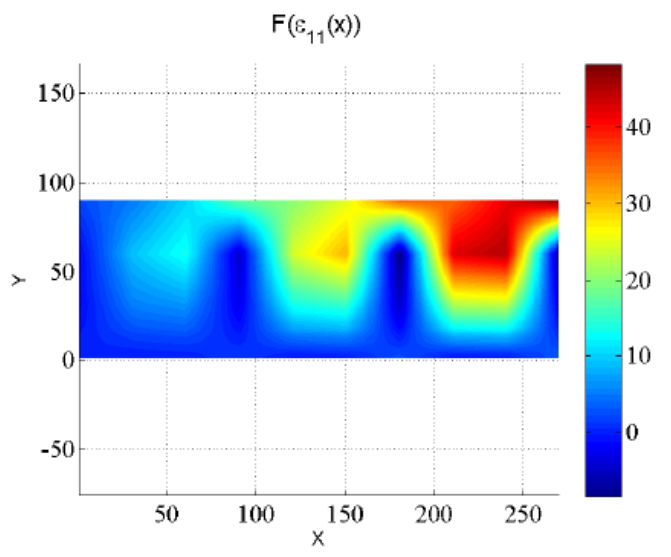

(b)

FIG. 7: (a) Reference solution for mesoscopic strain field $\mathcal{F}\left(\varepsilon_{11}^{\text {ref }}(\mathrm{x})\right)=\hat{\varepsilon}(\mathrm{x})$; (b) Reconstructed solution $\mathcal{F}\left(\varepsilon_{11}(\mathrm{x})\right)$ from the coarse-grained model.

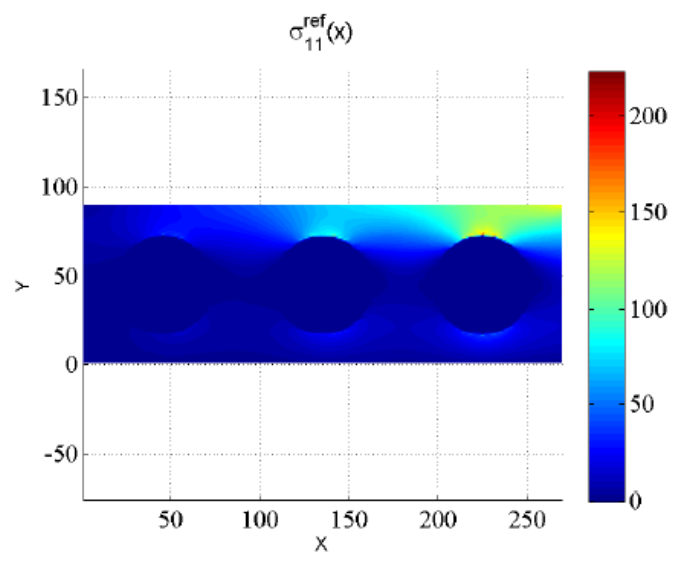

(a)

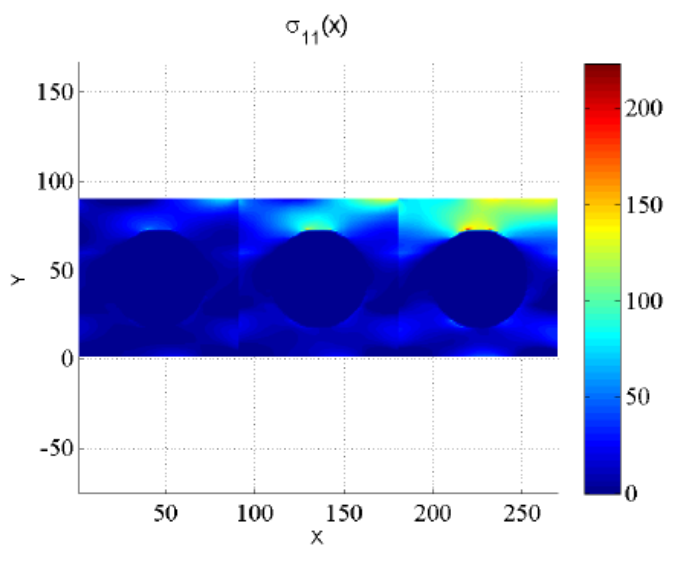

(b)

FIG. 8: (a) Reference solution for local stress field $\sigma_{11}^{r e f}(\mathrm{x})$; (b) Reconstructed solution from the coarse-grained model.

In contrast with classical nonlocal models, the present one also involves a dependence on the position of the point where the stresses are computed, and allows capturing heterogeneous mesoscopic fields arising from the underlying microstructure, illustrating the heterogeneous nonlocal character of the present scheme. Furthermore, the presence of holes can be handled naturally.

\subsection{Microindentation}

In this second example, the problem of indentation of a heterogeneous structure is considered, when the characteristic dimensions of the heterogeneities are of comparable size with the load fluctuation length, here the contact length. The domain is a squared domain of dimensions $H \times H, H=360 \mu \mathrm{m}$, as shown in Fig. 10(a). The microstructure is 


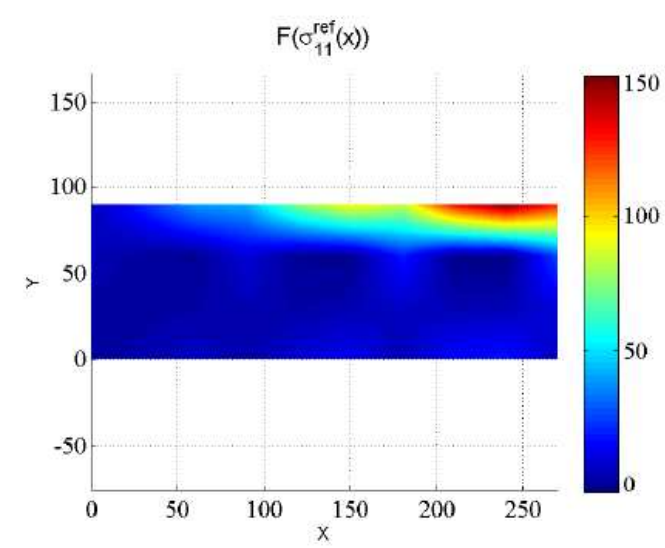

(a)

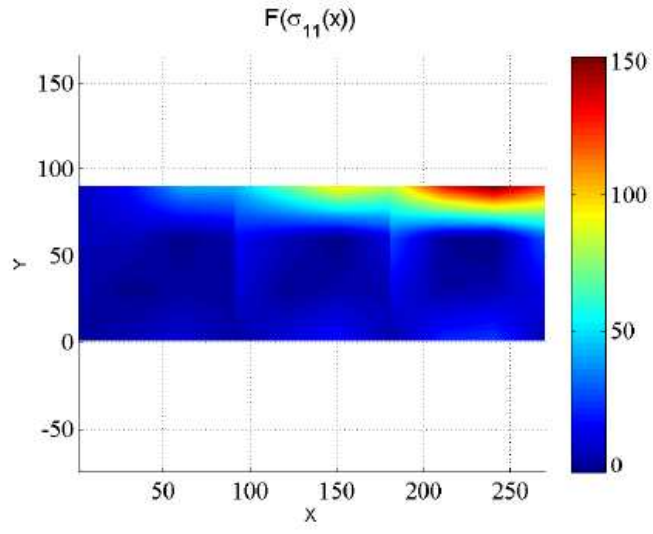

(b)

FIG. 9: (a) Reference solution for mesoscopic strain field $\mathcal{F}\left(\sigma_{11}^{r e f}(\mathrm{x})\right)$; (b) Reconstructed solution from the coarsegrained model.

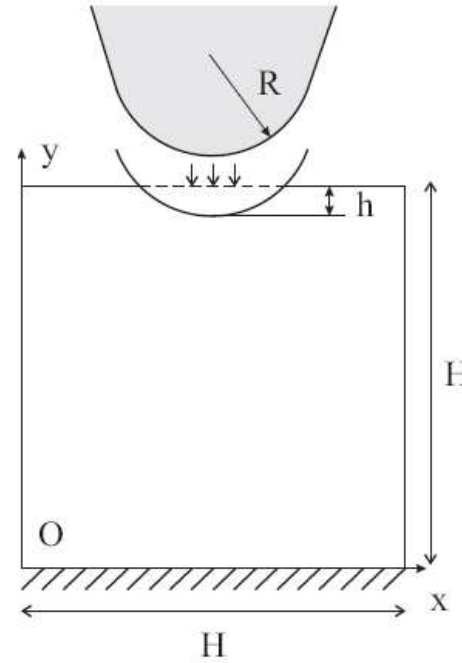

(a)

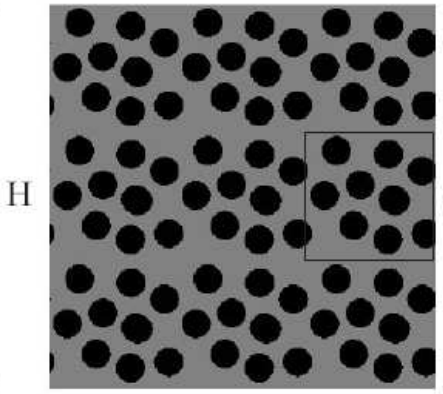

(b)

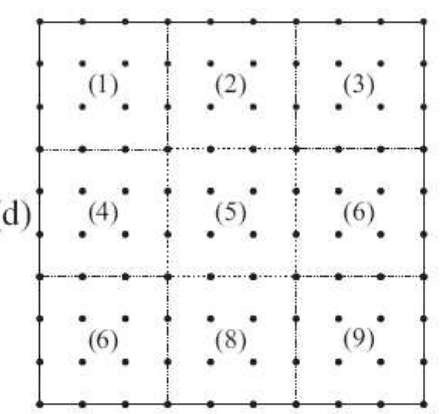

(c)

FIG. 10: (a) Geometry of the indentation problem; (b) microstructure; (c) coarse grid and subdomains; (d) unit cell.

composed by $3 \times 3$ periodic cells, each containing 9 circular inclusions with volume fraction $f=0.3$, as depicted in Fig. 10(b). The unit cell is depicted in Fig. 10(d). The indenter is modeled as a circular rigid surface of radius $R=$ $156 \mu \mathrm{m}$. Its penetration depth in the domain is $h=H / 30$.

The material properties of the matrix are $\lambda_{\text {mat }}=1 \mathrm{GPa}, \mu_{\text {mat }}=1 \mathrm{GPa}$. Two cases are treated in the following. The first case corresponds to rigid inclusions, with elastic properties $\lambda_{\text {mat }}=10 \mathrm{GPa}, \mu_{\text {mat }}=10 \mathrm{GPa}$. The second case corresponds to voids, modeled as soft inclusions with properties $\lambda_{\text {mat }}=10^{-3} \mathrm{GPa}, \mu_{\text {mat }}=10^{-3} \mathrm{GPa}$.

The lower end of the structure $(y=0)$ is fixed. To model the contact with the indenter, the points at the upper end $(y=H)$ which are within the circular portion of the indenter are projected along $y$ over the surface of the circle and corresponding Dirichlet boundary conditions are prescribed for these nodes along $y$. 
The mesh is composed of regular 4-node bilinear elements and $360 \times 360$ nodes. A $10 \times 10$ coarse grid is associated with the structure for defining the mesoscopic model [see Fig. 10(c)]. The mesh associated with the unit cell is regular with $120 \times 120$ nodes. Computations of the mesoscopic localization and effective tensors are computed on the unit cell with a coarse $4 \times 4$ grid.

For the two cases, corresponding to rigid inclusions and pores, the validation procedure is the same as in the previous example. The reference mesoscopic strain field $\mathcal{F}\left(\varepsilon_{i j}^{r e f}(\mathrm{x})\right)$ corresponding to each subdomain (1)-(9) [see Fig. 10(c)] is prescribed over the unit cell. Using the nonlocal homogenization procedure, the local and mesoscopic strain and stress fields within the unit cell corresponding to each subdomain are determined by post-treatment using (3.2), (3.18), and (3.19). Results of Figs. 11-14(b) and 15-18(b) show the reconstruction of the fields within the unit cell for each mesoscopic strain field in the subdomains (1)-(9).

Comparison with the reference solution is provided in Figs. 11-14 for rigid inclusions and in Figs. 15-18 for the porous structure. This example presents a much more localized mesoscopic strain field and the agreement between the local strain fields and the reference local strain fields is not as good as in the previous example. However, despite differences in local fluctuations, the structures of the fields and the orders of magnitude are similar, which is remarkable, taking into account the low number of cells involved in the process. From a general point of view, the reconstructed mesoscopic strain fields compare very well with the reference solution. More pronounced differences are noticed for the stress fields, for both local and mesoscopic fields. We can notice also that the reconstructed solution induces some discontinuities between the solutions in each subdomain. However, in the case of classical homogenization, the fields would be also discontinuous, as the mesoscopic fields would then be constant within each mesoscopic element. These results show the promising potential of the present framework.

\subsection{Mesoscopic Models of a Unit Cell from the Microscopic Scale to the Macroscopic Scale}

In this example, we illustrate the capability of the present scheme to continuously construct homogenized mesoscopic models for characteristic length scales ranging from the finest scale fluctuation length up to the case of separated scales. For this purpose, a unit cell whose microstructure is depicted in Fig. 19(a) is considered. This microstructure is assumed to be representative of a periodic porous material whose pores are organized into clusters. The pores have radii $R 2=0.075 \mathrm{~L}$ and the cluster is a circular region of radius $R 1=0.6 L$, with $L=1 \mathrm{~mm}$ the length of the square domain containing the microstructure, leading to a porosity $f=0.353$. As previously, the pores are modeled with very compliant properties: $\lambda_{i n c}=10^{-3}=\mu_{i n c}=10^{-3} \mathrm{GPa}$, while the properties of the matrix are

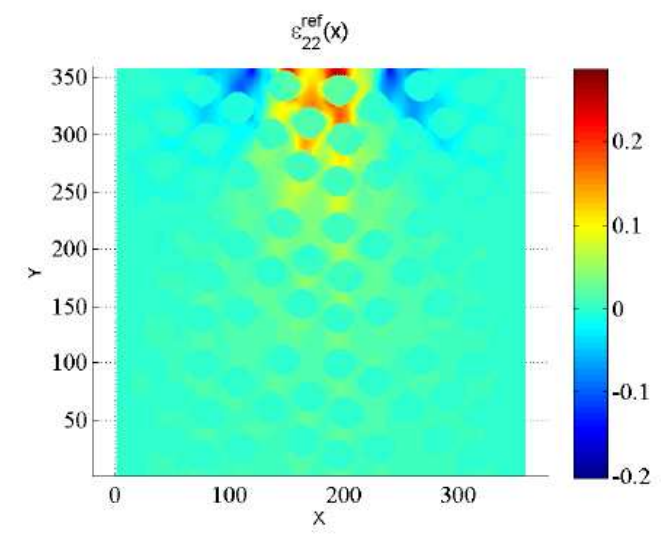

(a)

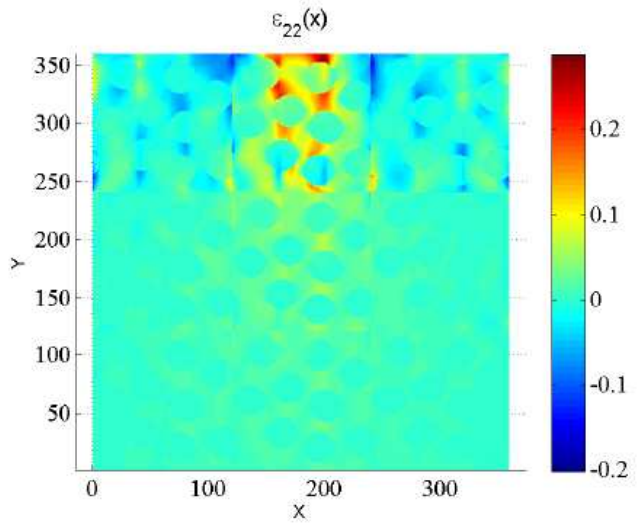

(b)

FIG. 11: (a) Rigid inclusions: reference solution for the local strain field $\varepsilon_{22}^{\text {ref }}(\mathbf{x})$; (b) Reconstructed solution $\varepsilon_{22}(\mathrm{x})$ from the coarse-grained model. 


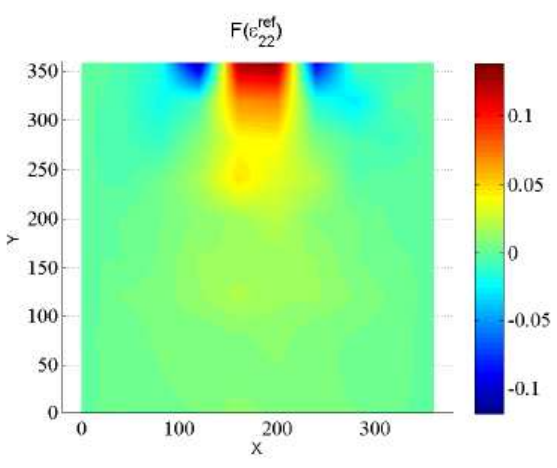

(a)

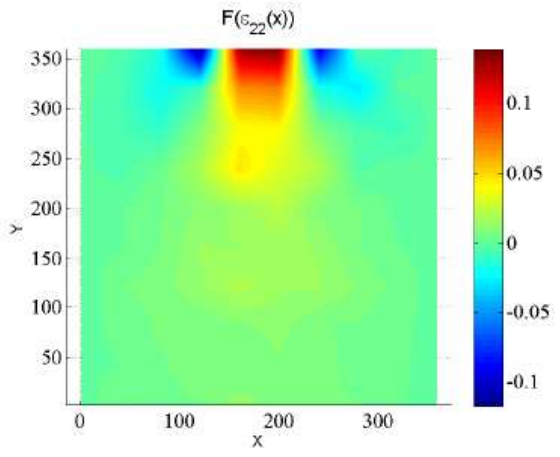

(b)

FIG. 12: (a) Rigid inclusions: reference solution for mesoscopic strain field $\mathcal{F}\left(\varepsilon_{22}^{r e f}(\mathrm{x})\right)=\hat{\varepsilon}_{22}(\mathrm{x})$; (b) Reconstructed solution $\mathcal{F}\left(\varepsilon_{22}^{r e f}(\mathrm{x})\right)$ from the coarse-grained model.

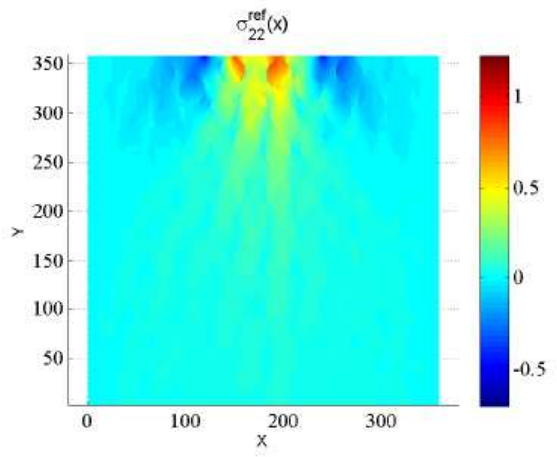

(a)

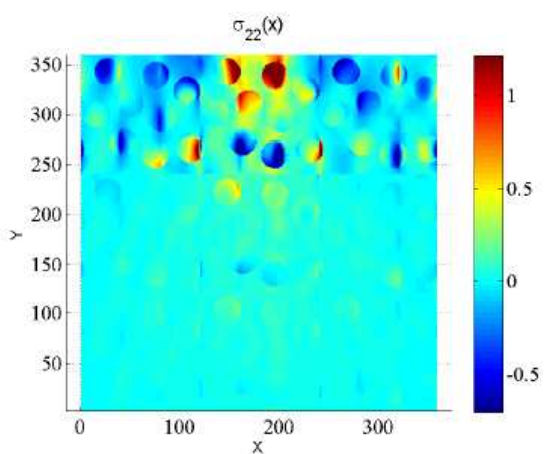

(b)

FIG. 13: (a) Rigid inclusions: reference solution for local stress field $\sigma_{22}^{r e f}(\mathrm{x})$; (b) Reconstructed solution $\sigma_{22}(\mathrm{x})$ from the coarse-grained model.

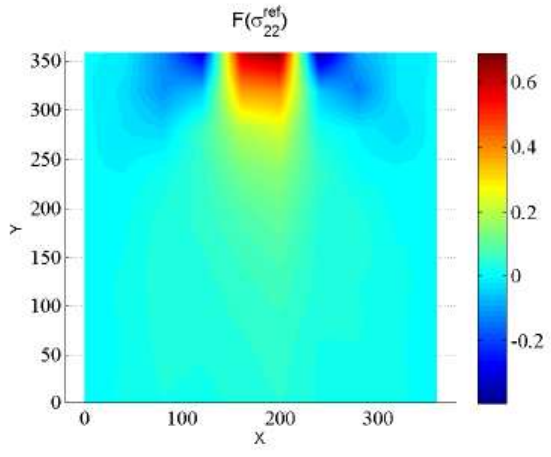

(a)

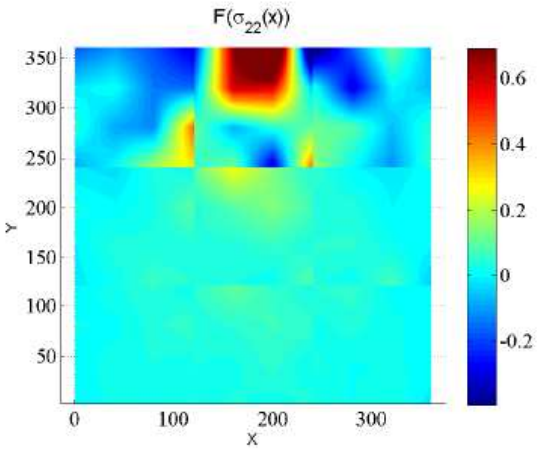

(b)

FIG. 14: (a) Rigid inclusions: reference solution for local stress field $\mathcal{F}\left(\sigma_{22}^{r e f}(\mathrm{x})\right)$; (b) Reconstructed solution $\sigma_{22}(\mathrm{x})$ from the coarse-grained model. 


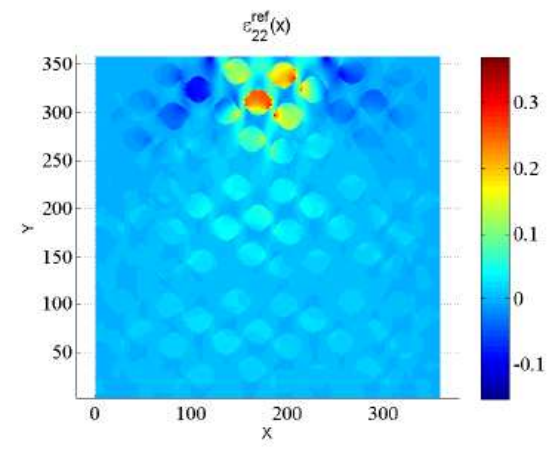

(a)

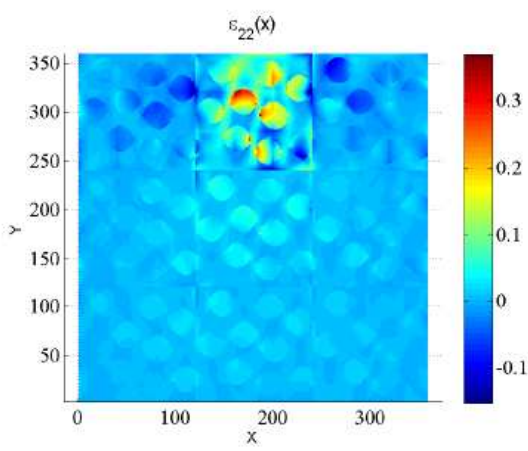

(b)

FIG. 15: (a) Porous structure: reference solution for the local strain field $\varepsilon_{22}^{r e f}(\mathbf{x})$; (b) Reconstructed solution $\varepsilon_{22}(\mathrm{x})$ from the coarse-grained model.

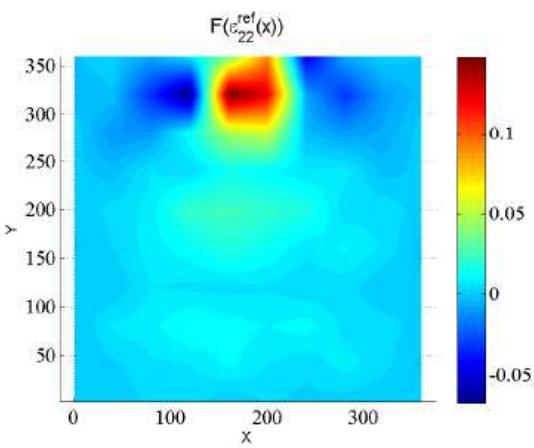

(a)

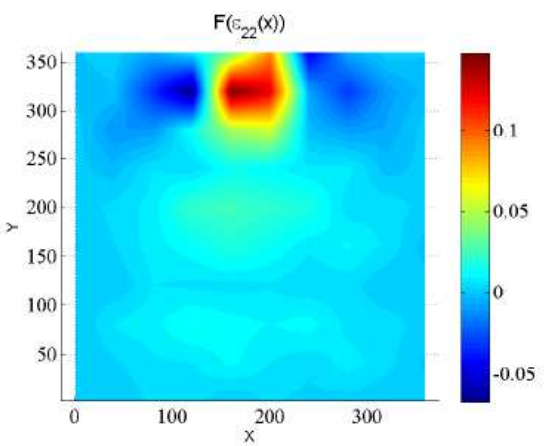

(b)

FIG. 16: (a) Porous structure: reference solution for mesoscopic strain field $\mathcal{F}\left(\varepsilon_{22}^{\text {ref }}(\mathrm{x})\right)=\hat{\varepsilon}_{22}(\mathrm{x})$; (b) Reconstructed solution $\mathcal{F}\left(\varepsilon_{22}^{\text {ref }}(\mathrm{x})\right)$ from the coarse-grained model.

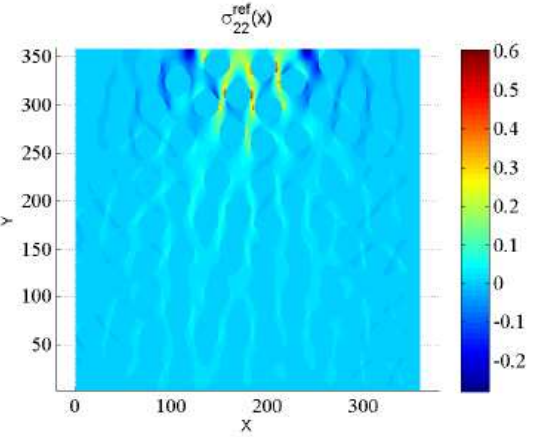

(a)

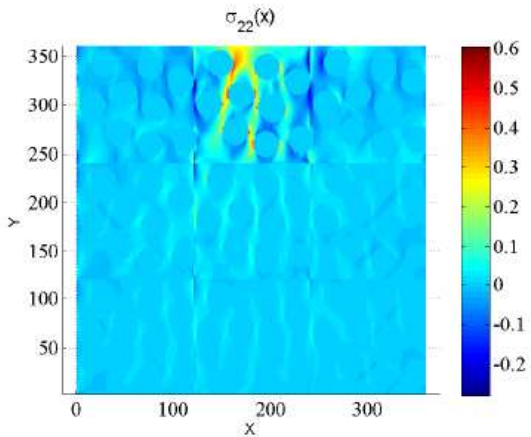

(b)

FIG. 17: (a) Porous structure: reference solution for local stress field $\sigma_{22}^{r e f}(\mathrm{x})$; (b) Reconstructed solution $\sigma_{22}(\mathrm{x})$ from the coarse-grained model. 


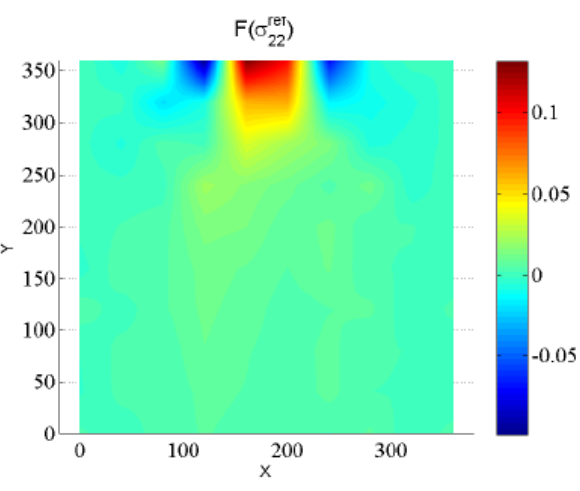

(a)

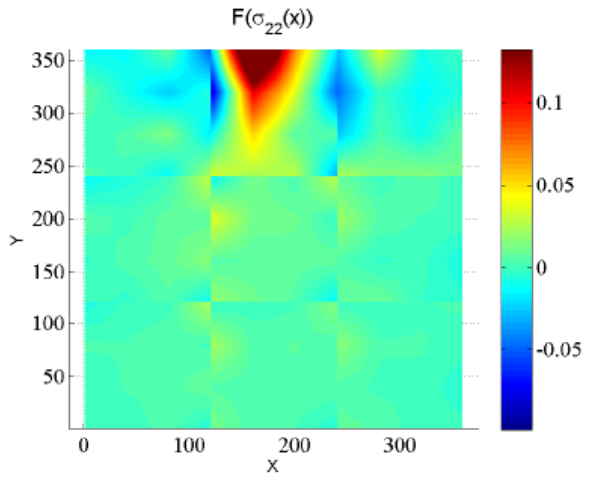

(b)

FIG. 18: (a) Porous structure: reference solution for local stress field $\mathcal{F}\left(\sigma_{22}^{r e f}(\mathrm{x})\right)$; (b) Reconstructed solution $\sigma_{22}(\mathrm{x})$ from the coarse-grained model.

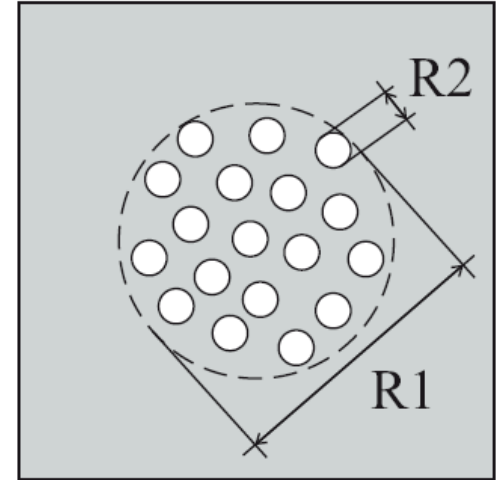

(a)

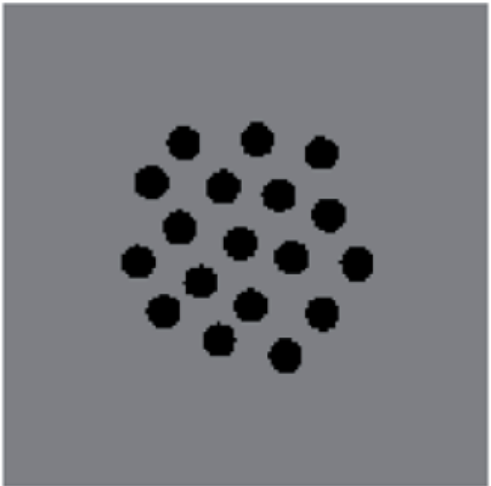

(b)

FIG. 19: Microstructure with clusters of pores: (a) Geometry; (b) mesh.

$\lambda_{\text {mat }}=1=\mu_{\text {mat }}=1 \mathrm{GPa}$. The mesh associated with the fine scale is composed of $144 \times 144$ regular 4-node elements. We apply the present homogenization framework for several filters, each associated with a characteristic wavelength $h$. Denoting by $\alpha$ the fine mesh element size, the following cases are considered: (a) $h / \alpha=1 ;$ (b) $h / \alpha=$ 12 ; (c) $h / \alpha=24$; (d) $h / \alpha=48$; (e) $h / \alpha=144$, and (f) $h / \alpha \gg 1$ (separated scales). The different intermediate cases correspond to the following number of $P \times P$ nodes on the coarse grid: (b) $13 \times 13$; (c) $7 \times 7$; (d) $4 \times 4$, and (e) $2 \times 2$. For each case, the different nonlocal effective tensors $\hat{\mathbb{C}}^{p}(\mathrm{x})$ are computed according to the procedure described in Section 3.2. At the finest scale, a reference solution is defined by subjecting the microstructure to a homogeneous strain field $\bar{\varepsilon}=\mathbf{v}_{1} \otimes \mathbf{v}_{1}$, leading to a strain field denoted by $\varepsilon^{r e f}(\mathbf{x})$. For each case $h / \alpha$, the mesoscopic strain field is computed by $\hat{\varepsilon}(\mathrm{x})=\mathcal{F}^{h}\left(\varepsilon^{r e f}(\mathrm{x})\right)$ and prescribed on the nodes of the coarse grid to compute the mesoscopic stress through Eq. (3.2). The microscopic stress solution is depicted in Fig. 20, and the homogenized solution is depicted in Fig. 22(b). The different intermediate mesoscopic solutions related to the different fluctuation length ration $h / \alpha$ are depicted in Figs. 20(a) and 22(b). 


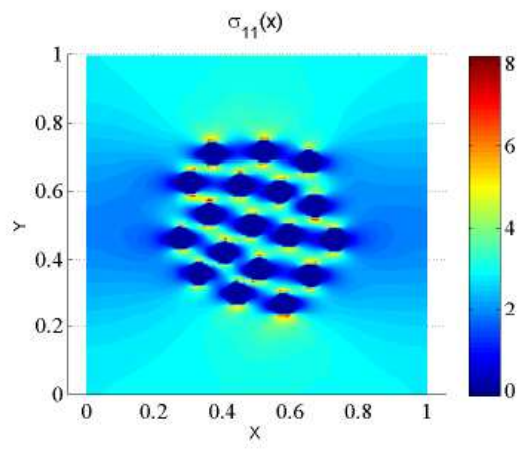

(a)

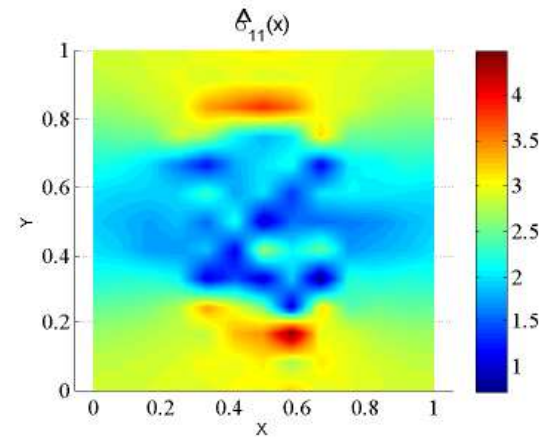

(b)

FIG. 20: (a) Microscopic $\sigma_{11}(\mathrm{x})$ stress solution, $h / \alpha=1$; (b) Computed mesoscopic stress solution $\hat{\sigma}_{11}(\mathrm{x})$ for $h / \alpha$ $=12, P \times P=13 \times 13$.

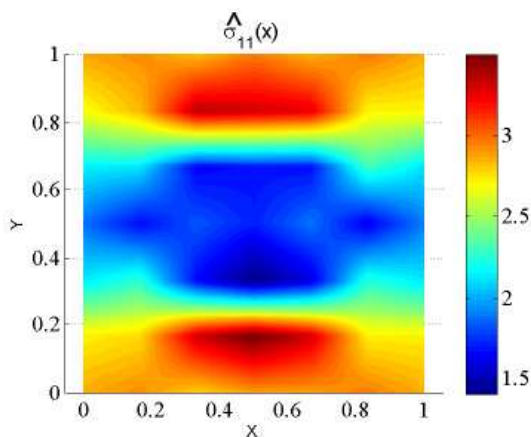

(a)

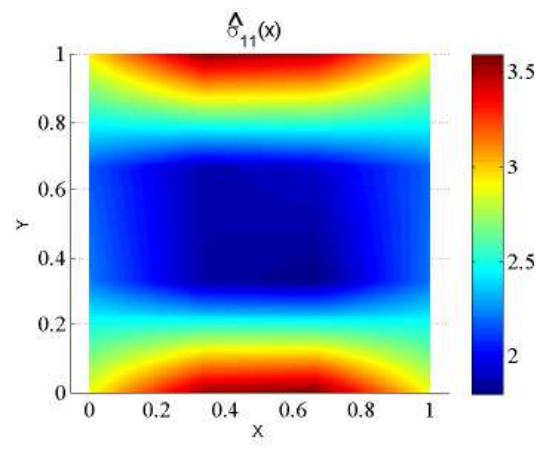

(b)

FIG. 21: (a) Computed mesoscopic stress solution $\hat{\sigma}_{11}(\mathrm{x})$ for $h / \alpha=24, P \times P=7 \times 7$; (b) Computed mesoscopic stress solution $\hat{\sigma}_{11}(\mathrm{x})$ for $h / \alpha=48, P \times P=4 \times 4$.

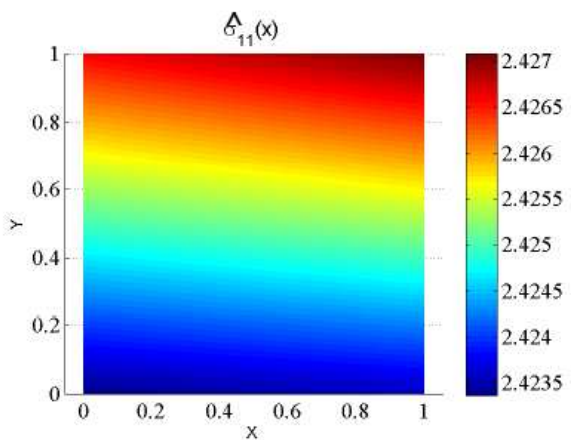

(a)

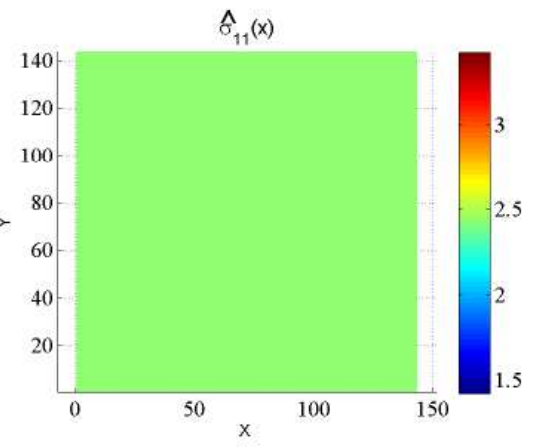

(b)

FIG. 22: (a) Computed mesoscopic stress solution $\hat{\sigma}_{11}(\mathrm{x})$ for $h / \alpha=144, P \times P=2 \times 2$; (b) Homogenized stress solution $\hat{\sigma}_{11}(\mathrm{x})$ for the classical case of separated scales. 


\section{CONCLUSION}

In this paper, we have introduced a new computational homogenization method for dealing with heterogeneous materials in the case of nonseparated scales, i.e., when the characteristic length of heterogeneities is no more negligible as compared to the one of load variations. The theoretical framework is an extension of classical homogenization, in which averaging operators are replaced by filter operators and localization operators by Green's operators defined over the unit cell. In the present paper, the filtering process is based on a projection of the fine scale fluctuations over a finite element basis of shape functions with characteristic spacing associated with the mesoscopic strain field. As a result, an equivalent mesoscopic (coarse-grained) media can be defined, where the constitutive law appears to be naturally nonlocal. An associated finite element methodology has been defined to compute the different mesoscopic constitutive and relocalization operators from a unit cell associated with the microstructure. This methodology includes the solution of a nonlocal problem by using an iterative process. The numerical examples, involving structures with heterogeneities (pores and rigid inclusions), show that an equivalent mesoscopic model can accurately reproduce both stress and strain fields with respect to the filtered fields related to a reference solution. Furthermore, as the methodology is fully micromechanically based, and in contrast to most nonlocal elasticity approaches, the localization tensors allow reconstructing all local strain and stress fields in the unit cell, for an arbitrary given mesoscopic strain field. Such a mesoscopic model is promising for upscaling heterogeneous materials in the case of nonseparated scales and constitutes a way for coarse-graining continuum heterogeneous materials in a systematic manner, to save computational times by several order of magnitude with respect to a fully detailed (meshed) model. Future developments will include a full FEM procedure to solve the structure problem directly at the mesoscopic scale, which involves the nonlocal constitutive equations.

\section{REFERENCES}

Bacigalupo, A. and Gambarotta, L., Non-local computational homogenization of periodic masonry, J. Multiscale Comput. Eng., vol. 9, no. 5, pp. 565-578, 2011.

Beran, M. and McCoy, J., Mean field variations in a statistical sample of heterogeneous linearly elastic solids, Int. J. Solids Struct., vol. 4, pp. 1035-1054, 1970.

Boutin, C., Microstructural effects in elastic composites, Int. J. Solids Struct, vol. 33, no. 7, pp. 1023-1051, 1996.

Bouyge, F., Jasiuk, I., and Ostoja-Starzewski, M., A micromechanically based couple-stres model of an elastic orthotropic twophase composite, Int. J. Solids Struct, vol. 38, pp. 1721-1735, 2001a.

Bouyge, F., Jasiuk, I., and Ostoja-Starzewski, M., A micromechanically based couple-stres model of an elastic two-phase composite, Int. J. Solids Struct., vol. 38, pp. 1721-1735, 2001b.

Coenen, E., Kouznetsova, V., and Geers, M., Multi-scale continuous-discontinuous framework for computational-homogenizationlocalization, J. Mech. Phys. and Solids, vol. 60, pp. 1486-1507, 2012.

Duarte, C. and Kim, D.-J., Analysis and applications of a generalized finite element method with global-local enrichment functions, Comput. Methods Appl. Mech. Eng., vol. 197, pp. 487-504, 2008.

Eringen, A. and Edelen, D., On nonlocal elasticity, Int. J. Eng. Sci., vol. 10, pp. 233-243, 1972.

Fish, J., Jiang, T., and Yuan, Z., A staggerd nonlocal multiscale model for a heterogeneous medium, Int. J. Numer. Methods Eng., vol. 91, pp. 142-157, 2012.

Forest, S., Mechanics of generalized continua: construction by homogenizaton, J. Phys. IV France, vol. 8, pp. 39-48, 1998.

Forest, S., Dendievel, R., and Canova, G., Estimating the overall properties of heterogeneous cosserat materials, Model. Simul. Mater. Sci. Eng., vol. 7, pp. 829-840, 1999.

Forest, S. and Sab, K., Cosserat overall modelling of heterogeneous materials, Mech. Res. Commun., vol. 25, no. 4, pp. 449-454, 1998 .

Gao, J., An asymetric theory of nonlocal elasticity. Part 2. Continuum field, Int. J. Solids Struct., vol. 36, pp. 2959-2971, 1999.

Geers, M., Coenen, E., and Kouznetsova, V., Multi-scale cmputational homogenization of structured thin sheets, Model. Simul. Mater. Sci. Eng., vol. 15, pp. 393-404, 2007. 
Giacco, G., Zerbino, R., Ponce, J., and Batic, O., Mechanical behavior of concrete damaged by alkali-silica reaction, Cement Conrete Res., vol. 38, pp. 993-1004, 2009.

Hui, T. and Oskay, C., A nonlocal homogenization model for wave dispersion in dissipative composite materials, Int. J. Solids Struct., vol. 50, pp. 38-48, 2013.

Kouznetsova, V., Geers, M., and Brekelmans, W., Constitutive modeling of heterogeneous materials with gradient enhanced computational homogenization scheme, Int. J. Numer. Methods Eng., vol. 54, pp. 1235-1260, 2002.

Kouznetsova, V., Geers, M., and Brekelmans, W., Multi-scale second-order computational homogenization of multi-phase materials: a nested finite element solution strategy, Comput. Methods Appl. Mech. Eng., vol. 193, pp. 5525-5550, 2004.

Kroner, E., Elasticity theory of materials with long-range cohesive forces, Int. J. Solids Struct., vol. 3, pp. 731-742, 1967.

Kruch, S. and Forest, S., Computation of coarse grain structures using a homogeneous equivalent medium, J. Phys. IV France, vol. 8, pp. 197-205, 1998.

Mindlin, R., Micro-structure in linear elasticity, Arch. Rational Mech. Anal., vol. 16, pp. 51-78, 1964.

Mindlin, R. and Eshel, N., On first gradient theory in linear elasticity, Int. J. Solids Struct., vol. 4, pp. 109-124, 1968.

Moës, N., Cloirec, M., Cartraud, P., and Remacle, J.-F., A computational approach to handle complex microstructure geometries, Comput. Methods Appl. Mech. Eng., vol. 192, pp. 3163-3177, 2003.

Moulinec, H. and Suquet, P., A fast numerical method for computing the linear and nonlinear properties of composites, $C$. $R$. l'Académie des Sciences, vol. 318, pp. 1417-1423, 1994.

Ostoja-Starzewski, M., Boccara, S., and Jasiuk, I., Couple-stress moduli and characteristic length of a two-phase composite, Mech. Res. Commun., vol. 26, no. 4, pp. 387-396, 1999.

Peerlings, R., deBorst, P., Brekelmans, W., de Vree, J., and Spee, I., Some observations on localization in non-local and gradient damage models, Eur. J. Mech. A/Solids, vol. 15, no. 6, pp. 937-953, 1996.

Polizzotto, C., Nonlocal elasticity and related variational principles, Int. J. Solids Struct., vol. 38, pp. 7359-7380, 2001.

Porubov, A., Aero, E., and Maugin, G., Two approaches to study essentially nonlinear and dispersive properties of the internal structure of materials, Phys. Rev. E, vol. 79, pp. 1216-1243, 2009.

Smyshlyaev, V. and Cherednichenko, K., On rigorous derivation of strain gradient effects in the overall behaviour of periodic heterogeneous media, J. Mech. Phys. Solids, vol. 48, pp. 1325-1357, 2000.

Toupin, R., Theories of elasticity with couple-stress, Arch. Rat. Mech. Anal., vol. 11, no. 1, pp. 385-414, 1964.

Tran, T.-H., Monchiet, V., and Bonnet, G., A micromechanics-based approach for the derivation of constitutive elastic coefficients of strain-gradient media, Int. J. Solids Struct., vol. 49, pp. 783-792, 2012.

Willis, J., The overall elastic response of composite materials, J. Appl. Mech., vol. 50, pp. 1202-1209, 1983.

Yuan, X. and Tomita, Y., A micromechanical approach of nonlocal modeling for media with periodic microstructures, Mech. Reas. Commun., vol. 35, pp. 126-133, 2008.

Yvonnet, J., A fast method for solving microstructural problems defined by digital images: A space Lippmann-Schwinger scheme, Int. J. Numer. Methods Eng., vol. 92, no. 2, pp. 178-205, 2012.

Yvonnet, J. and Bonnet, G., A consistent nonlocal scheme based on filters for the homogenization of heterogeneous linear materials with non-separated scales, Int. J. Solids Struct., vol. 51, pp. 196-209, 2014. 